\title{
Platelet Estimation by Manual and Automated Methods
}

\author{
M Lavanya ${ }^{1}$, C Jayanthi ${ }^{1 *}$, Alexandria Maria ${ }^{2}$ and V Janani ${ }^{1}$ \\ ${ }^{1}$ Sri Venkateshwaraa Medical College Hospital and Research Centre \\ ${ }^{2}$ Sri ManakulaVinayagar Medical college Hospital
}

\begin{abstract}
Background: Accurate platelet estimation is essential for the management of the patients with thrombocytopenia. Automated analyzer by its quick and reliable platelet estimation has overtaken the manual methods. But sometimes they do produce erroneous results which are to be cross checked by manual methods.

Hence this study was undertaken to compare the traditional manual method and alternate method based on Platelet/RBC ratio for estimating platelets in peripheral smear and to find the most accurate method for detecting Thrombocytopenia.

Methods: 100 samples of EDTA anticoagulated blood is analysed by automated analyzer, then thin peripheral smear is prepared. Manual counts are performed by 1: Traditional Method- average platelet count / 10 Oil immersion field (OIF) x 15,000, 2: Alternate method -platelet/1000 red blood cells (RBC) multiplied by RBC count. Manual methods were compared with the automated analyzer using students t test. The Sensitivity, Specificity, Positive Predictive Value (PPV), Negative Predictive Value (NPV) and Likelihood ratio (LR) for positive tests were also calculated for the manual and alternate methods in detecting thrombocytopenia.

Result: The students t test shows no difference in the platelet counts estimated by traditional method and alternate method. Further the results of sensitivity, specificity, PPV, NPV and LR for positive tests show the traditional and Alternate method can give fairly accurate results.

Conclusion: The manual method of platelet estimation is found to be the most accurate and can used to quality check the automated analyzer or can be used in underprivileged laboratories, for aiding in proper management of the patient.
\end{abstract}

\section{Keywords: Manual Platelet Count, Platelet/RBC Ratio, Peripheral Smear, Diagnostic Accuracy}

\section{Introduction}

Platelets are small, anucleate cytoplasmic fragments present in blood, which play a key role in hemostasis and thrombosis. Platelet counts tend to be the least reproducible of the blood cell counts, because they are small and have a tendency to adhere to glass, any foreign body, and particularly to one another.$^{[1]}$ Accurate and reproducible platelet counts is essential for patient management. Normal range of platelet count in a healthy individual is $150-400$ $\mathrm{x} 10^{3} / \mu \mathrm{L}^{[2-4]}$

Platelet count can be estimated by various methods including manual methods (Hemocytometer counting with phase contrast microscopy, peripheral blood smear) and by automated methods [2,3]. Manual methods are time consuming, subjective and tedious with high levels of imprecision. Now a days the automated haematology analyser with quick and accurate complete blood count has replaced the traditional manual methods[1].

Automated hematology analyzers sometimes produce erroneous results that do not match with the clinical condition of the patient especially in thrombocytopenia.
Standard guidelines with each instrument mandate performing manual platelet counts below and above the established reference ranges[1].

The International Council for Standardization in Hematology (ICSH) and the International Society of Laboratory Hematology (ISLH) have recommended a method based on the measurement of platelet/RBC ratio with fluorescent labeled platelets in fluorescent flow cytometer as the reference method for platelet counting in peripheral blood[10,11,12]. But this method is expensive and cannot be performed routinely in developing countries[10,11,13].

Hence this study is planned to compare the platelet count estimated by traditional manual methods with the alternate method based on Platelet/ RBC ratio.

\section{Materials And Methods}

This is a cross sectional comparative study done in Department of Pathology, Central Laboratory, Sri Venkateshwaraa Medical College and Research Centre, Puducherry from July 2017 to August 2017. The study has been approved by the Scientific Research Committee and 
waiver of consent obtained from the Institutional Ethical Committee (Human studies). 100 blood samples received in the Hematology Laboratory in K2 EDTA vacutainer for complete hemogram were included for the study. The samples which were inadequate, clotted or lysed and smears with platelet clumps and satellitism were excluded from the study.

The collected samples were analyzed within half an hour of collection, in the automated hematology analyser MINDRAY (BC-5150) 5 part which estimates the platelet count by impedence method and the complete blood count report were obtained. The name and age of the patient were noted along with the RBC count(millions $/ \mathrm{mm}^{3}$ ) and platelet count (lakhs $/ \mathrm{mm}^{3}$ ).

The peripheral smears were prepared by wedge method and stained with Leishman stain. The slides were examined under microscope in oil immersion and platelets were counted in the area where the red cells are just touching one another without overlapping. The platelet count is calculated as follows.

Average number of platelets / Oil Immersion Field (OIF) multiplied by 15000 (Traditional method) [5]

The number of platelets per 1000 RBC multiplied by RBC count (Alternate method ) [6]

Samples with low $\left(<1.5\right.$ lakhs $\left./ \mathrm{mm}^{3}\right)$ and normal counts (1.5- 4 lakhs $/ \mathrm{mm}^{3}$ ) on automated analyzer were taken and the manual and alternate methods were compared for detecting thrombocytopenia. Samples with high counts were excluded.

\section{Statistical Analysis}

The data was entered in Microsoft Excel and analyzed using SPSS software version 23.0. The variables such as age and platelet count are expressed as mean (standard deviation). The Spearman correlation and Student $t$ test was done. $\mathrm{P}$ value of $<0.05$ is considered statistical significance. For calculating the sensitivity, specificity, positive predictive value (PPV), negative predictive value (NPV) and Likelihood ratio (LR) for positive tests, the platelet counts with low (20 numbers) and normal counts (67 numbers) were taken.

\section{Result}

Total 100 samples were analysed. The age distribution was between 1 and 85 years (Median age - 45 years). The $\mathrm{RBC}$ count ranged from 1.22 to 7 million / cu.mm .The platelet count ranged from 14 to $697 \times 10^{3} / \mu \mathrm{L}$ with mean of $251.26 \times 10^{3} / \mu \mathrm{L}$.

58 samples were from males and 42 samples from females with mean platelet count of $213.57( \pm 120.16) \times 10^{3} / \mu \mathrm{L}$ and $303.31( \pm 121.22) \times 10^{3} / \mu \mathrm{L}$ respectively. The student $t$ test showed significant difference in the mean platelet values of male and females (p- 0.0001).

The platelet count range, mean values and standard deviationestimated by automated, traditional method and alternate method are given in Table 1.

The difference between the traditional and alternate methods were calculated by the Students T test which did not showstatistically significant difference (Table 2).

The accurate method of platelet estimation in detecting thrombocytopenia is found by calculating the sensitivity, specificity, positive predictive value (PPV), negative predictive value (NPV) and Likelihood Ratio (LR). The results of these tests are given in Table 3 .

\section{Discussion}

Estimation of platelet count by automated method has taken over the manual methods because of quick, accurate and reliable estimation of platelet count with coefficient of variation $(\mathrm{CV})$ of $<2 \%$. [8] The $\mathrm{CV}$ of manual hemocytometer methods range from $11-15 \%$, depending on number of platelets counted [8].

Table 1: Platelet estimation by Automated and Manual Methods.

\begin{tabular}{|l|c|c|c|}
\hline Methods & Platelet count range $\left(\mathbf{x} 10^{3} / \mu \mathrm{L}\right)$ & Mean Platelet count $\left(\mathbf{x} 10^{3} / \mu \mathrm{L}\right)$ & Standard deviation \\
\hline Automated analyzer & $14-697$ & 251.26 & 127.98 \\
\hline Traditional Method & $21-552$ & 234.99 & 114.65 \\
\hline Alternate Method & $21.3-723$ & 267.56 & 143.67 \\
\hline
\end{tabular}

Table 2: Comparison of manual methods with automated analyzer.

\begin{tabular}{|c|c|c|}
\hline Statistical test & Traditional Method & Alternate Method \\
\hline Student t test (p value) & 0.3448 (NS) & 0.3979 (NS) \\
\hline
\end{tabular}

NS- Not Significant

S-Significant 
Table 3: Accuracy of Manual methods in detecting Thrombocytopenia.

\begin{tabular}{|l|c|c|}
\hline Statistical test & Traditional Method & Alternate Method \\
\hline Sensitivity (\%) & 90 & 85 \\
\hline Specificity(\%) & 94.03 & 95.5 \\
\hline Positive predictive value(\%) & 81.8 & 85 \\
\hline Negative predictive value(\%) & 96.9 & 95.5 \\
\hline Likelihood Ratio for positive test & 15.08 & 18.9 \\
\hline
\end{tabular}

However, the Automated analyzers sometimes produce erroneous results. Fragments of leucocyte cytoplasm can falsely elevate the count. Falsely low counts occur in platelet satellitism (platelets adhering to neutrophils), clumping due to agglutinins, spontaneous aggregation, or incipient clotting due to faulty blood collection $[1,4,8]$. Hence each instrument comes with standard guidelines to mandate performing manual platelet counts below and above the established reference ranges[1].

The manual methods of estimating platelet counts are done: 1) using Hemocytometer 2) Examination of Peripheral blood Smear. In the Hemocytometer method blood is diluted with various diluents like $1 \%$ ammonium oxalate $[1,8]$ or brilliant cresyl blue (Rees Ecker method) [9]can be used with a phase contrast microscopy.

In peripheral blood method, on average, if the platelet count is normal, about one platelet is found per 10-30 red cells. At 1000 magnification, this is equivalent to about 7-20 platelets per oil immersion field in the areas where red cell morphology is optimal [1]. This has led to the traditional method of calculating platelets by taking an average of platelet count in 10 oil immersion fields multiplied by 15000 in case of venous blood, and 20000 in capillary blood [9]. The higher multiplication factor in capillary blood may be due to platelets adhering at the site of skin puncture.

The alternate methods are based on Platelet / $\mathrm{RBC}$ ratio [6] and the inverse relationship of hemoglobin with platelet count [7] respectively.

The International Council for Standardization in Hematology (ICSH) and the International Society of Laboratory Hematology (ISLH) have recommended a method based on the measurement of platelet/RBC ratio with fluorescent labeled platelets in fluorescent flow cytometer as the reference method for platelet counting in peripheral blood[10,11,12]. But this method is expensive and cannot be performed routinely in developing countries[10,11,13].

In our study, we found no difference between the mean platelet count estimated by traditional method and the automated analyzer, similar to study done by Bajpai et al[2] and Webb et al [5]. But studies done by Sudalaimuthu et al [10] and Momodu[14] show significant difference between the two methods. Hence a study has to be conducted with a large sample size to find the significance of traditional method of platelet estimation. In our laboratory, we use platelet estimation by traditional method whenever the automated analyser gives a low count. We find the tradition method reliable in the management of thrombocytopenia.

Studies done by Momodu and Anitha et al $[14,15]$ comparing the traditional and automated method show no difference, but they have used the multiplication factor 20,000 instead of 15000 used in our study.

The alternate method based on the Platelet/ RBC ratio and automated method show no significant difference in our study and other studies done by Umarani et al, Brahmini et al and Bhayal et al $[2,6,11]$. So this method can be considered as alternate reference method.

Both Traditional method and Alternate method show no statistically significant difference with the automated analyzer, however Alternate Method with high sensitivity, specificity, PPV and Likelihood ratio for positive test is the most accurate manual method in the detection of thrombocytopenia (Table 3).

\section{Conclusion:}

Alternate method of platelet estimation by platelet/RBC ratio can be used as a reference Manual Method, but the traditional manual method can still be considered as gold standard for use in rural settings or in small labs where sophisticated equipments are not available. The manual methods can also be used to quality check the automated instruments whenever the values are erratic and outside the normal range.

\section{Acknowledgements}

I sincerely thank Dr. S. Srikanth, Professor of Community Medicine for helping in the statistical analysis of our study.

\section{Reference}

1. Neerja V, Susan S G, Sylva B. Basic examination of blood and bone marrow. In: Richard A. McPherson, Matthew R. 
Pincus, editors. Henry's clinical diagnosis and management by laboratory methods.22nd ed. Philadelphia: Elsiever Saunders; 2011. pp 515-531.

2. Bajpai, et al. Platelet count by peripheral blood smear: Reliable, rapid, cost-effective method to assess degree of thrombocytopenia. International Journal of Medical Science Research and Practice.2015; Vol 2 ( 2): 90-93.

3. Umarani MK, Shashidhar B. Estimation of platelet count from peripheral blood smear based on platelet: red blood cell ratio. A prospective study in a tertiary care hospital. Indian Journal of Pathology and Oncology, April-June 2016;3(2);351-353

4. Ninama NJ, Nirali K Shah. Impedance platelet count in severe microcytosis-study of 161 patients. NHL Journal of Medical Sciences. Jan 2014;Vol 3( 1):32-36.

5. Webb DI, Parker L, Webb K. Platelet count assessment from peripheral blood smears (PBS).Alaska Med. 2004 OctDec;46(4):92-5.

6. Brahimi et al. Platelet count from a blood smear. Turk J Hematol 2009; 26: 21-4.

7. Malok M et al. Comparison of two platelet count estimation methodologies for peripheral blood smears. Clin Lab Sci. 2007 Summer;20(3):154-60.

8. Sherrie L P. Examination of the Blood and Bone Marrow. In: John P. Greer, John Foerster, George M. Rodgers, Frixos Paraskevas, Bertil Glader, Daniel A. Arber, Robert T. Means Jr, editors. Wintrobe's Clinical Hematology.12th ed. Philadelphia: Lippincott Williams \& Wilkins; 2009. pp. 9-46.

9. Cheryl B, M.S. Peripheral blood smear. In: Shirlyn B. McKenzie, Mark Cohen, Melissa Kerian editor. Clinical Laboratory Hematology. New Jersey: Pearson Education.

10. Sudalaimuthu $\mathrm{M}$ et al. A novel method to estimate platelet counts from peripheral smears: A study comparing a new method of platelet estimation with existing methods. Annals of Pathology and Laboratory Medicine, Jan - Feb 2017; Vol. 04( 01); 77-82.

11. Dr. Ajamal S B et al. Study of platelet count on the basis of Red cell: Platelet ratio. JMSCR. Oct 2015; Volume 03 (10); 7742-45.

12. Bakhubaira S. Automated Versus Manual Platelet Count in Aden. Clinical \& Experimental Pathology.2013. vol 3 (3)

13. Babadoko AA, Ibrahim IN, Musa AU, Usman $\mathrm{N}$. Reproducibility of hematological parameters: Manual versus automated method. Sub-Saharan Afr J Med. 2016; 3:65-70.

14. Imoru M. Determination of platelet and white blood cell counts from peripheral blood smear: an indispensable method in under-resourced laboratories. International Blood Research and Reviews.2016; 5(2): 1-7.

15. Anitha $\mathrm{K}$ et al. Comparison of Platelet Count by Peripheral Smear Method and Automated Method in pregnant women. National Journal of Physiology, Pharmacy \& Pharmacology,2014; Vol 4 ( 1 ); 39 - 42.

*Corresponding author:

Dr. Jayanthi C2, Assistant professor, Department of Pathology, Sri Manakula Vinayagar Medical college and Hospital, Pondicherry, India

Phone: +91 9994323960

Email: drjayanthichandran@gmail.com

Financial or other Competing Interests: None. 